\title{
Effect of tea catechins on postprandial plasma lipid responses in human subjects
}

\author{
Tomonori Unno ${ }^{1 *}$, Motomi Tago ${ }^{2}$, Yuko Suzuki ${ }^{1}$, Ayumu Nozawa ${ }^{1}$, Yuko M Sagesaka ${ }^{1}$, Takami Kakuda ${ }^{1}$, \\ Kazutada Egawa $^{3}$ and Kazuo Kondo ${ }^{2}$ \\ ${ }^{1}$ Central Research Institute, ITO EN, Ltd., 21 Mekami, Sagara-cho, Haibara-gun, Shizuoka 421-0516, Japan \\ ${ }^{2}$ Institute of Environmental Science for Human Life, Ochanomizu University, 2-1-1 Ohtsuka, Bunkyo-ku, Tokyo 112-8610, Japan \\ ${ }^{3}$ Nihonbashi Egawa Clinic, 1-1-3 Yaesu, Chuo-ku, Tokyo 103-0028, Japan
}

(Received 3 August 2004 - Revised 1 November 2004 - Accepted 19 November 2004)

\begin{abstract}
Epidemiological surveys suggest that a higher intake of tea may be associated with a lower risk of CHD. There is accumulating evidence that postprandial lipaemia makes a substantial contribution to the incidence of CHD. Our aim was, therefore, to evaluate the effect of tea catechins (major ingredients in green tea) on postprandial lipid responses in human subjects after the consumption of test meals. In a randomized triple-crossover design, nine male subjects with mild or borderline hypertriacylglycerolaemia consumed 10 (control), 224 (moderate dose) and 674 mg (high dose) of the assigned tea catechins three times each along with a standardized light meal consisting of a piece of bread spread with $20 \mathrm{~g}$ butter. Plasma lipids were measured in the fasting state and $1,2,3$, 4 and $6 \mathrm{~h}$ after consuming the light meal. Results showed that, compared with the control, moderate and high doses of tea catechins reduced the incremental area under the plasma triacylglycerol curves by 15.1 and $28.7 \%$, respectively. Next, the rapid elevation of remnant-like particle cholesterol was significantly inhibited by a high dose of tea catechins $2 \mathrm{~h}$ after consuming the light meal $(P<0 \cdot 01)$. In the range of tea catechin dosages, no significant differences were observed in the postprandial responses for plasma total cholesterol or NEFA at any time point. In conclusion, this trial demonstrated that tea catechins attenuated the postprandial increase in plasma triacylglycerol levels following a fat load. These results may provide evidence for one of the possible mechanisms involved in lowering the incidence of CVD, and may prove useful in further studies on the beneficial health effects of tea drinking.
\end{abstract}

Tea catechins: Triacylglycerols: Remnant lipoprotein: Postprandial lipaemia

A great deal of scientific interest has been aroused in the beneficial health effects of tea (Camellia sinensis). The most extensively investigated benefit is its role in preventing CHD (Yang \& Landau, 2000; Peters et al. 2001; Kris-Etherton \& Keen, 2002; McKay \& Blumberg, 2002). Epidemiological surveys provide evidence that tea consumption may be associated with lowering CHD mortality rates (Tijburg et al. 1997; Hollman et al. 1999; Peters et al. 2001).

Several lines of evidence suggest that elevated levels of fasting plasma triacylglycerols may be an independent risk factor for CVD (Jeppesen et al. 1998). Over the last decade, a few studies have been conducted on the effects of tea catechins on the fasting plasma triacylglycerols in animal models (Matsumoto et al. 1998; Vinson \& Dabbagh, 1998). The proposed mechanism underlying the hypolipidaemic activity of tea catechins is most likely mediated by its influence on the intestinal absorption of dietary fat (Chan et al. 1999). Moreover, a Japanese cross-sectional study reported that an increased consumption of green tea was associated with a lower fasting serum triacylglycerol level (Imai \& Nakachi, 1995).
In recent years, however, there has been a renewal of interest in whether the postprandial elevation of plasma triacylglycerols may be more closely related to atherogenic risk rather than fasting triacylglycerol levels (Sanders, 2003). In this respect, the primary treatment for reducing the risk of CHD is to follow a low-fat diet, while another possible treatment is to ingest something that selectively limits the intestinal absorption of dietary fat. Recent attention has been paid to the latter possibility. Some studies demonstrated that red wine polyphenols attenuate postprandial plasma lipid response in human subjects, possibly by delaying the absorption of dietary fat through the intestine (Kondo et al. 1998; Pal et al. 2004). As already stated, tea is a rich source of catechin polyphenols. Although an animal study demonstrated that intestinal fat absorption was significantly decreased in rats orally administered a single dose of (-)-epigallocatechin gallate, which is a major component in tea catechins (Raederstorff et al. 2003), the clinical contribution of tea catechins to the intestinal absorption of dietary fat is not so far understood.

The aim of the present study was to evaluate the effect of tea catechins on the postprandial response of plasma triacylglycerols after an oral fat load on human subjects. 


\section{Methods}

\section{Test beverages}

A semi-purified catechin extract from green tea (THEA-FLAN 90S; ITO EN, Ltd, Tokyo, Japan) was used for the preparation of test beverages. This extract was rich in (-)-epigallocatechin gallate and (-)-epicatechin gallate. The present study employed three dose challenges of the following tea catechins in total: (1) $10 \mathrm{mg}$ tea catechins as a control; (2) $224 \mathrm{mg}$ as a moderate dose; (3) $674 \mathrm{mg}$ as a high dose. Table 1 gives the contents of individual tea catechins per bottle serving $(250 \mathrm{ml})$ of each test beverage. In addition to the tea catechins, other ingredients such as cyclodextrin, L-ascorbic acid and a green tea-flavoured catechin-free liquid were added to the respective beverages at the same levels as the control.

\section{Subjects}

The clinical aspects of this study were proposed in accordance with the Helsinki Declaration of 1964 as revised in 1989, and were approved by the Ethical Committee of the Nihonbashi Egawa Clinic (Tokyo, Japan). Subjects were recruited from the Nihonbashi Egawa Clinic. The procedures were explained in detail to all subjects in advance, and all gave their informed written consent for participation in the study. Exclusion criteria included chronic ailments such as hepatic, renal or cardiac dysfunction, and malabsorption. Individuals who were on medication or consuming health foods that may affect the lipid metabolism were not enrolled in this study. Based on screening blood tests carried out within 2 weeks, nine male adults with borderline and mild hypertriacylglycerolaemia (range $1.38-2.49 \mathrm{mmol} / \mathrm{l}$ ) were sampled. Physical characteristics and fasting metabolic profiles of the participants are shown in Table 2. Subjects were required to abstain from any alcoholic beverages for $2 \mathrm{~d}$ before each visit.

\section{Experimental protocol}

Upon entry into the study, subjects were randomly divided into three groups. Each group was assigned to one of three treatment sequences to ensure a study conducted in a double-blind-controlled manner. The three testing sessions were separated by 1 -week

Table 1. Tea catechin composition of the test beverages $(\mathrm{mg} / 250 \mathrm{ml})^{*}$

\begin{tabular}{lccr}
\hline & \multicolumn{3}{c}{ Catechin dose } \\
\cline { 2 - 4 } & Control & Moderate & High \\
\hline Tea catechins (total) & 10 & 224 & 674 \\
(-)-Epigallocatechin gallate & 1 & 68 & 243 \\
(-)-Gallocatechin gallate & 0 & 88 & 233 \\
(-)-Epicatechin gallate & 0 & 33 & 118 \\
(-)-Catechin gallate & 1 & 30 & 70 \\
(-)-Epigallocatechin & 1 & 0 & 0 \\
(-)-Gallocatechin & 5 & 5 & 5 \\
(-)-Epicatechin & 1 & 0 & 5 \\
(-)-Catechin & 1 & 0 & 0 \\
\hline
\end{tabular}

*A tea catechin extract (THEA-FLAN 90S; ITO EN, Ltd, Tokyo, Japan) was used for preparing test beverages (moderate and high). THEA-FLAN 90S mainly contains $47 \%$ of $(-)$-epigallocatechin gallate and $15 \%$ of $(-)$-epicatechin gallate of the dry weight. In the manufacturing process of green tea beverages, a part of such catechin is epimerized into $(-)$-gallocatechin gallate and $(-)$-catechin gallate.
Table 2. Subject characteristics and fasting plasma lipid levels at screening (Mean values with their standard errors for nine subjects)

\begin{tabular}{lccc}
\hline & Mean & SE & \multicolumn{1}{c}{ Range } \\
\hline Age (years) & 46 & 3 & $(33-63)$ \\
Body weight $(\mathrm{kg})$ & 78 & 8 & $(65-88)$ \\
BMI $\left(\mathrm{kg} / \mathrm{m}^{2}\right)$ & 26.8 & 0.8 & $(23.6-30.6)$ \\
Triacylglycerols $(\mathrm{mmol} / \mathrm{l})$ & 1.98 & 0.14 & $(1.38-2.49)$ \\
Total cholesterol $(\mathrm{mmol} / \mathrm{l})$ & 5.77 & 0.38 & $(4.11-7.44)$ \\
HDL-cholesterol $(\mathrm{mmol} / \mathrm{l})$ & 1.17 & 0.07 & $(0.91-1.51)$ \\
NEFA (mEq/l) & 0.44 & 0.04 & $(0.32-0.72)$ \\
\hline
\end{tabular}

intervals. In between sessions, the subjects were free to continue their usual lifestyle, eating and exercise habits. On the evening before an experiment, the subjects ate a standardized light dinner before 21.00 hours to avoid the effects of previous food intake, and then refrained from all food and beverages (except water) until 09.00 hours on the following morning. After blood was taken from the antecubital vein under overnight fasting conditions, each subject consumed the fat-enriched bread and test beverage within $10 \mathrm{~min}$. The test meal consisted of one piece of sliced white bread (70 g) (Yamazaki Baking Co. Ltd, Tokyo, Japan) and butter ( $20 \mathrm{~g}$ ) (Snow Brand Milk Products, Co. Ltd, Sapporo, Japan), the total nutrient composition of which was calculated as $7.0 \mathrm{~g}$ protein, $18.8 \mathrm{~g}$ fat and $31.8 \mathrm{~g}$ carbohydrate (total energy $1364 \mathrm{~kJ})$. Blood samples were collected 1, 2, 3, 4 and $6 \mathrm{~h}$ after the completion of the light meal. No food (except $300 \mathrm{ml}$ water) was allowed until the end of the study.

\section{Biochemical analyses}

Plasma was separated from whole blood by centrifuging at $1200 \mathrm{~g}$ for $20 \mathrm{~min}$, and subjected to biochemical analyses of triacylglycerols, total cholesterol and NEFA using automatically enzymebased methods. Remnant-like particle (RLP) cholesterol was determined with a JIMRO-II assay kit (Japan Immunoresearch Laboratories, Takasaki, Japan) using an immunoseparation method.

\section{Statistics}

Results are expressed as means with their SEM. The increment areas under the time $v$. concentration curves (increment area under the curves, IAUC) were evaluated after subtracting the initial individual values $(0 \mathrm{~h})$ from all respective postprandial measurements, yielding the net postprandial change as calculated by the trapezoidal rule (Matthews et al. 1990). Comparisons between results from the test beverages were made by repeatedmeasures ANOVA followed by Dunnett's test. A value of $P<0.05$ was considered statistically significant. These statistical calculations were performed with GraphPad Prism for Windows version 4.0 (GraphPad Software, San Diego, CA, USA).

\section{Results}

All participants were able to follow the study protocol without difficulty. No adverse effects from the experimental beverages were reported by subjects during or after the testing sessions. There were no significant differences in the baseline levels 
of plasma triacylglycerols, total cholesterol and NEFA among the three different sessions (data not shown).

The incremental postprandial responses of plasma triacylglycerols are illustrated in Fig. 1(A). When the subjects consumed the test meal with a control beverage, plasma triacylglycerol concentrations quickly rose from the baseline $(0 \mathrm{~h})$, and reached their highest levels $3 \mathrm{~h}$ after the test-meal intake. Concentrations did not return to baseline for $6 \mathrm{~h}$. Clearly, the peak plasma triacylglycerols were reduced by co-administrations of tea catechins in a dose-dependent manner. Repeated-measures one-way ANOVA with post hoc comparisons using Dunnett's test applied to the data showed that, compared with control, the plasma triacylglycerol response to the oral fat load was significantly lower 2 and $3 \mathrm{~h}$ after a high dosage of tea catechins $(P<0.05$, both ways $)$. This was reflected in the significant effect of tea catechins from the high-dosage treatment on the mean IAUC as shown in Table $3(P<0 \cdot 05)$. Expressed as a percentage of control, the average reductions in IAUC after a moderate and a high-catechin dosage were 15.1 and $28.7 \%$, respectively. However, no significant effect on IAUC was observed between the control and the moderate dosage of tea catechins.
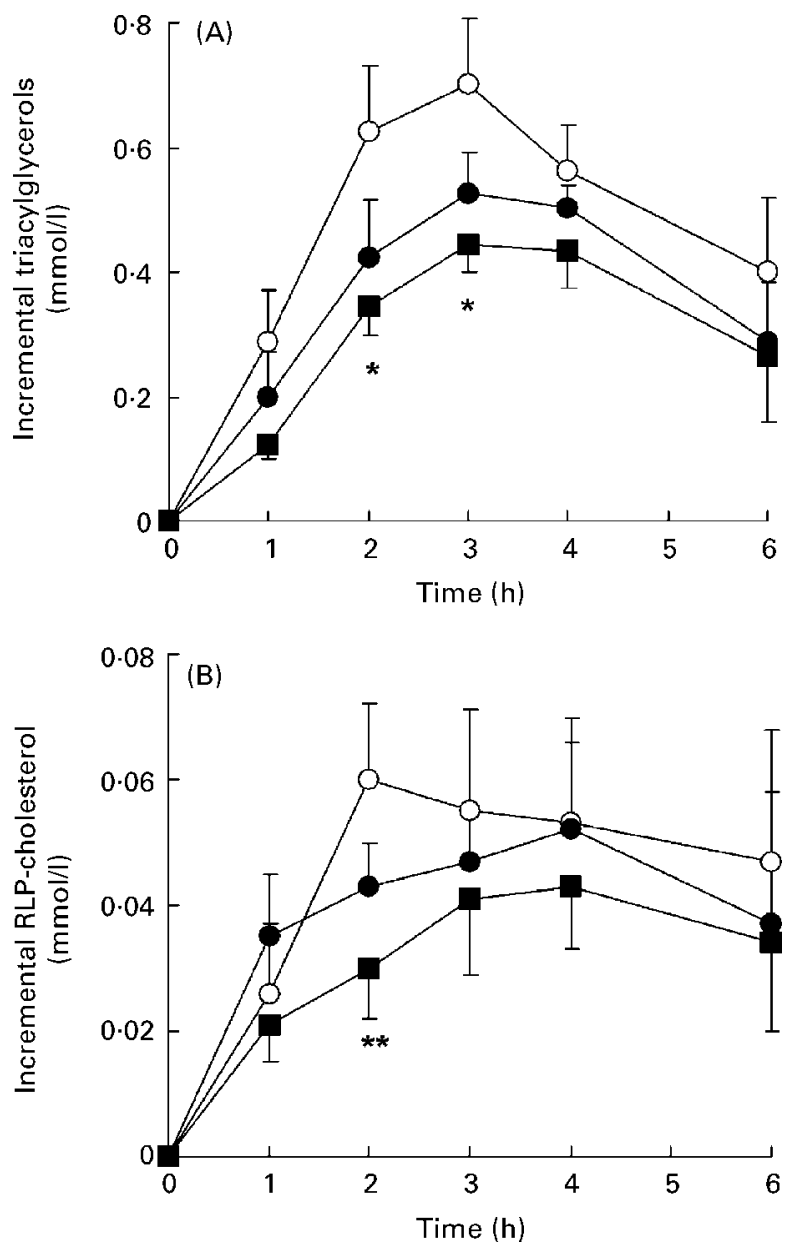

Fig. 1. Changes in plasma triacylglycerols $(A)$ and remnant-like particle (RLP)-cholesterol (B) after an oral administration of a butter-enriched light meal along with each of a control $(\bigcirc)$, moderate $(\bullet)$ and high $(\square)$ tea catechin-containing beverage. Values are means for nine subjects with their standard errors represented by vertical bars. Mean values were significantly different from those of the control group at each time point: ${ }^{\star} P<0.05$, ${ }^{\star \star} P<0.01$.
The same was true of the plasma level of RLP-cholesterol. Fig. 1(B) shows that the maximum level of plasma RLP-cholesterol was reached $2 \mathrm{~h}$ after consuming the test meal with a control beverage. With moderate and high dosages, however, the rise in plasma RLP-cholesterol continued for $4 \mathrm{~h}$. The rapid elevations in plasma RLP-cholesterol were suppressed by the tea catechin supplementations, with a statistically significant difference between the control and the high dosage at $2 \mathrm{~h}(P<0.01)$, whereas the repeated-measures ANOVA for detecting differences across treatments showed no statistically significant difference in IAUC among the three test beverages $(P=0 \cdot 10)$ (Table 3$)$.

There was a mild increase in plasma total cholesterol levels during the study period (Fig. 2(A)). Meanwhile, plasma-NEFA showed a temporary downturn from the baseline, and then turned upward (Fig. 2(B)). Postprandial levels of total cholesterol and NEFA were found to be unrelated to the different dosage levels of tea catechins at any time point.

\section{Discussion}

To date, a number of clinical studies have been conducted to evaluate the effect of tea consumption on the potential risk factors for CVD. Investigation of the cardiovascular-protective effects of tea can be focused on the prevention of oxidative damage of LDL, interference with the atherosclerotic inflammatory process, reduction of thrombosis, and the promotion of normal endothelial function (Kris-Etherton \& Keen, 2002). In keeping with the current attention being paid to the association of postprandial lipaemia with the increased risk factors for CHD, the present study was carried out to investigate whether tea catechins could modulate postprandial lipaemia using human subjects in a triple-crossover design.

The test meals used in the present study were low in fat $(20 \mathrm{~g}$ butter) relative to other alimentary studies that used more than $50 \mathrm{~g}$ butter to stimulate responses (Thomsen et al. 1999, 2003; Mattes, 2001). A recent study by Igarashi et al. (2003) recommended a newly designed oral fat-loading test with the clinical use of $20 \mathrm{~g}$ butter along with an enteral nutrition drink having a combined energy level of $553 \mathrm{kcal}$. Our test meals induced an average $0.70 \mathrm{mmol} / \mathrm{l}$ increase in plasma triacylglycerols over the baseline level at $3 \mathrm{~h}$ in the control beverage sessions. Under such feeding conditions, tea catechin supplements could have exerted suppressing effects on the postprandial elevations in plasma triacylglycerols in a dose-dependent manner, and on the high dosage of tea catechins accompanied by a statistically significant difference in IAUC of plasma triacylglycerols as compared with the control. The assumption that exaggerated lipaemia in the postprandial phase may be implicated in the early development of atherosclerosis is now widely accepted, but a further hypothesis has gained attention recently, i.e. that impaired remnant lipoproteins specifically promote atherosclerosis and CVD. Plasma triacylglycerols are clearly a measure of triacylglycerol-rich lipoproteins (chylomicrons and VLDL) of intestinal and hepatic origin. Remnant lipoproteins are formed in the bloodstream from these triacylglycerol-rich lipoproteins by lipoprotein lipases and to a lesser extent by hepatic lipases (Syvänne \& Taskinen, 1997; Jialal \& Devaraj, 2002; Schaefer, 2002). The issue of the remnant characteristics of certain lipoprotein fractions has recently provoked new interest due to the development of a technique for the determination of RLP-cholesterol in plasma. The RLP-cholesterol assay provides a valid measure of atherogenic triacylglycerol-rich lipoprotein remnants, especially in patients with metabolic abnormalities such as hyperlipidaemia, type II 
Table 3. Effects of tea catechins on incremental area under the curve following an oral fat load

(Mean values with their standard errors)

\begin{tabular}{|c|c|c|c|c|c|c|c|}
\hline & \multicolumn{2}{|c|}{ Control } & \multicolumn{2}{|c|}{ Moderate } & \multicolumn{2}{|c|}{ High } & \multirow[b]{2}{*}{$P$ value } \\
\hline & Mean & SE & Mean & SE & Mean & SE & \\
\hline Triacylglycerols (mmol h/l) & $2 \cdot 87$ & 0.32 & $2 \cdot 21$ & 0.17 & $1 \cdot 86^{*}$ & 0.20 & 0.036 \\
\hline Remnant-like particle-cholesterol (mmol h/l) & 0.28 & 0.05 & 0.26 & 0.03 & 0.20 & 0.03 & 0.100 \\
\hline
\end{tabular}

Mean value was significantly different from control: ${ }^{\star} P<0.05$ ( post hoc comparison with Dunnett's test).

†For differences across treatments (ANOVA).

diabetes mellitus and renal disorders (Twickler et al. 2004). Recently, the effect of diet intervention on postprandial plasma RLP-cholesterol levels has been evaluated extensively, with a conceptual design to promote a reduction of postprandial RLP-cholesterol concentrations by soya protein (Shige et al. 1998) and dietary fibre (Kondo et al. 2004). In the present study, treatment with tea catechin supplements also resulted in the suppression of RLPcholesterol at an early postprandial stage, but it failed to achieve
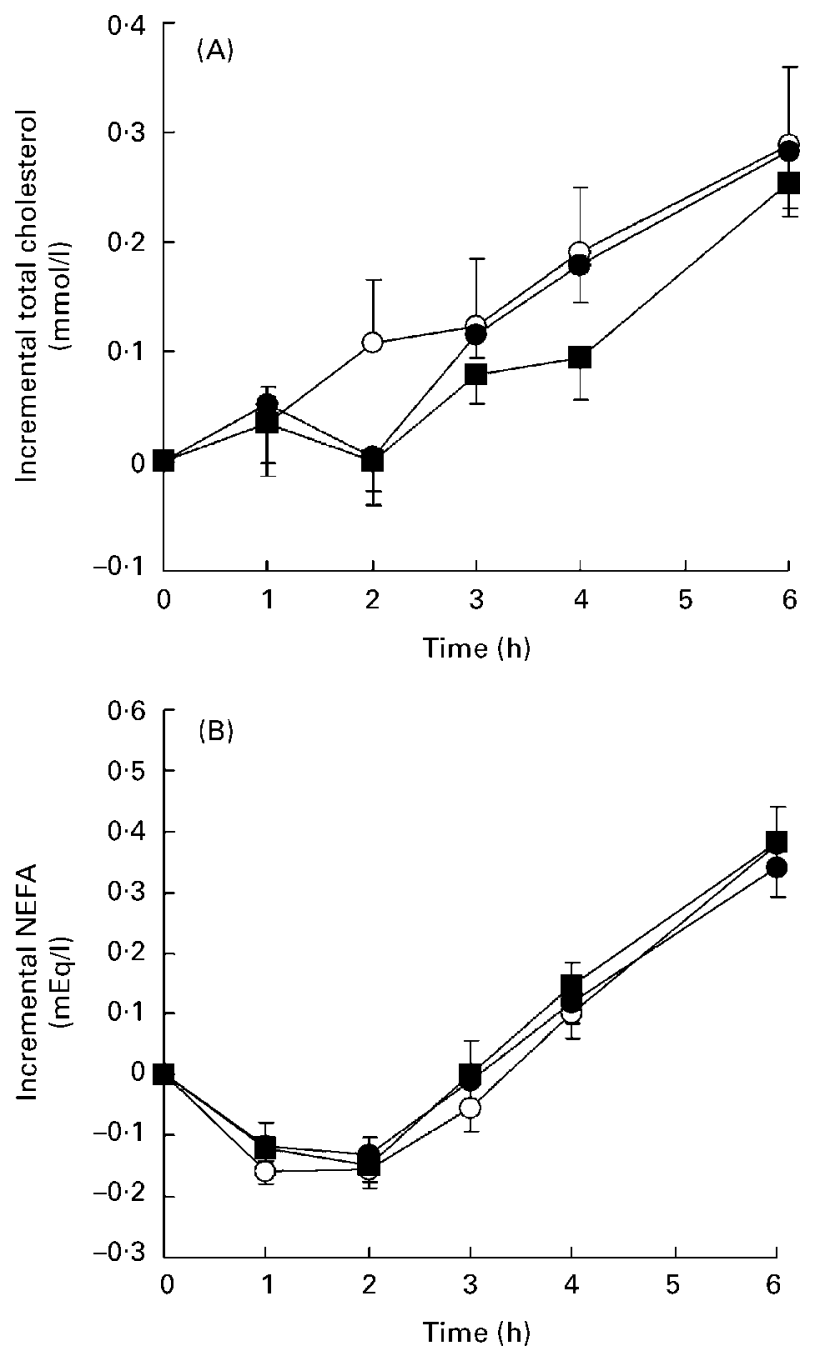

Fig. 2. Changes in plasma total cholesterol (A) and NEFA (B) after an oral administration of a butter-enriched meal along with each of a control $(O)$, moderate $(\bullet)$ and high $(\boldsymbol{\square})$ tea catechin-containing beverage. Values are means for nine subjects with their standard errors represented by vertical bars. statistical significance in IAUC of RLP-cholesterol. This seems likely to be due to the sample size of our subjects.

Although we did not elucidate in this study the mechanisms by which tea catechins attenuated postprandial plasma triacylglycerols, the work of others helps to account for our results. According to the in vitro study by Juhel et al. (2000), green tea extracts exhibited a marked ability to inhibit gastric and pancreatic lipases. It is well recognized that digestive lipases play key roles in lipid absorption through the intestinal tract to hydrolyse triacylglycerols to 2-monoacylglycerol and NEFA, both of which are incorporated into bile salt-phospholipid micelles, which in turn are absorbed into the brush border of the small intestine, eventually entering the bloodstream. In view of this, the assumption that tea catechins attenuate lipid absorption via digestive lipase inhibition at the in vivo stage may make sense. It should also be noted that factors such as differing lipid emulsification and droplet size affect intestinal lipid absorption. There is evidence to show that a smaller fat droplet size facilitates fat digestion by digestive lipases (Armand et al. 1999). A recent in vitro study showed that (- )-epigallocatechin gallate increased gradually in the scale of mixed micelles (Raederstorff et al. 2003). In this connection, it would be possible to argue that, although the evidence is not compelling, limited intestinal lipid absorption by tea catechins may contribute to postprandial plasma triacylglycerol responses at the in vivo stage (but the matter may be not quite as simple as this suggests).

One serving of the black tea consumed in the UK typically contains approximately $137-141 \mathrm{mg}$ total flavonoids per $235 \mathrm{ml}$ for $40-60 \mathrm{~s}$ of brewing time (Lakenbrink et al. 2000). Briefly, the amounts of tea catechins at moderate $(224 \mathrm{mg})$ and high $(674 \mathrm{mg})$ doses account for the equivalent of 1.6 and 4.8 cups, respectively. The point to keep in mind is that individual tea catechins such as $(-)$-epicatechin gallate, $(-)$-epigallocatechin gallate, (-)-catechin gallate and (-)-gallocatechin gallate, accounting for a large percentage of total tea catechins in the test beverages, exert strong inhibitory effects on the digestive lipases in vitro, whereas tea catechins such as (-)-epicatechin, $(-)$-epigallocatechin, (-)-catechin and (-)-gallocatechin have little effect (unpublished results). Provided that direct interaction between tea catechins and digestive lipases could occur in the gut, the choice of effective forms of tea catechins is of major importance in achieving a significant impact on postprandial lipaemia.

To our knowledge, this is the first controlled clinical trial to provide evidence confirming the suppressive effects of tea catechins on the postprandial levels of triacylglycerols and RLP-cholesterol. Given that tea is often consumed both during and after meals, such effects of tea catechins may, in fact, link to the epidemiological findings on the inverse association between tea drinking and CHD. However, an overall assessment of the beneficial effects of tea catechins ingested over long periods of 
time remains to be completed. Further studies are required to elucidate the long-term effects of tea catechins on lipid metabolism in human subjects as well as their detailed mechanisms of action.

\section{Acknowledgements}

Thanks are due to the staff of KSO Co. (Tokyo, Japan) for assistance in carrying out this study.

\section{References}

Armand M, Pasquier B, André M, Borel P, Senft M, Peyrot J, Salducci J, Portugal H, Jaussan V \& Lairon D (1999) Digestion and absorption of 2 fat emulsions with different droplet sizes in the human digestive tract. Am J Clin Nutr 70, 1096-1106.

Chan PT, Fong WP, Cheung YL, Huang Y, Ho WKK \& Chen Z (1999) Jasmine green tea epicatechins are hypolipidemic in hamsters (Mesocricetus auratus) fed a high fat diet. $J$ Nutr 129, 1094-1101.

Hollman PC, Fesken EJ \& Katan MB (1999) Tea flavonoids in cardiovascular disease and cancer epidemiology. Proc Soc Exp Biol Med 220, 198-202.

Igarashi M, Hirata A, Yamauchi T, et al. (2003) Clinical utility and approach to estimate postprandial hypertriglycemia by a newly designed oral fat-loading test. $J$ Atheroscler Thromb 10, 314-320.

Imai K \& Nakachi K (1995) Cross sectional study of effects of green tea on cardiovascular and liver diseases. Br Med J 310, 693-696.

Jeppesen J, Hein HO, Suadicani P \& Gyntelberg F (1998) Triglyceride concentration and ischemic heart disease. An eight-year follow-up in the Copenhagen male study. Circulation 97, 1029-1036.

Jialal I \& Devaraj S (2002) Remnant lipoproteins: measurement and clinical significance. Clin Chem 48, 217-219.

Juhel C, Armand M, Pafumi Y, Rosier C, Vandermander J \& Lairon D (2000) Green tea extract $\left(\operatorname{AR} 25^{\circledR}\right)$ inhibits lipolysis of triglycerides in gastric and duodenal medium in vitro. $J$ Nutr Biochem 11, 45-51.

Kondo K, Hosoda K, Iwamoto T, Kusumoto A, Hirano R, Matsumoto A \& Itakura H (1998) Red wine improves postprandial status. In XIIIth International Symposium on Drugs Affecting Liquid Metabolism $(D A L M)$, p. 33. Florence, Italy: Fondazione Giovanni Lorenzini Medical Science Foundation.

Kondo S, Xiao J, Takahashi N, Miyaji K, Iwatsuki K \& Kokubo S (2004) Suppressive effects of dietary fiber in yogurt on the postprandial serum lipid levels in healthy adult male volunteers. Biosci Biotechnol Biochem 68, $1135-1138$

Kris-Etherton PM \& Keen CL (2002) Evidence that the antioxidant flavonoids in tea and cocoa are beneficial for cardiovascular health. Curr Opin Lipidol 13, 41-49.

Lakenbrink C, Lapczynski S, Maiwald B \& Engelhardt UH (2000) Flavonoids and other polyphenols in consumer brews of tea and other caffeinated beverages. J Agric Food Chem 48, 2848-2852.
McKay DL \& Blumberg JB (2002) The role of tea in human health: an update. J Am Coll Nutr 21, 1-13.

Matsumoto N, Okushio K \& Hara Y (1998) Effect of black tea polyphenols on plasma lipids in cholesterol-fed rats. J Nutr Sci Vitaminol 44, $337-342$.

Mattes RD (2001) Oral exposure to butter, but not fat replacers elevates postprandial triacylglycerol concentration in human subjects. $J$ Nutr 131, 1491-1496.

Matthews JNS, Altman DG, Campbell MJ \& Royston P (1990) Analysis of serial measurements in medical research. $\mathrm{Br}$ Med $\mathrm{J} \mathbf{3 0 0}$, 230-235.

Pal S, Naissides M \& Mamo J (2004) Polyphenolics and fat absorption. Int J Obes 28, 324-326.

Peters U, Poole C \& Arab L (2001) Does tea affect cardiovascular disease? A meta-analysis. Am J Epidemiol 154, 495-503.

Raederstorff DG, Schlachter MF, Elste V \& Weber P (2003) Effect of EGCG on lipid absorption and plasma lipid levels in rats. $J$ Nutr Biochem 14, 326-332.

Sanders TAB (2003) Dietary fat and postprandial lipids. Curr Atheroscler Rep 5, 445-451.

Schaefer EJ (2002) Lipoproteins, nutrition, and heart disease. Am J Clin Nutr 75, 191-212.

Shige H, Ishikawa T, Higashi K, et al. (1998) Effects of soy protein isolate (SPI) and casein on the postprandial lipemia in normolipidemic men. $J$ Nutr Sci Vitaminol 44, 113-127.

Syvänne M \& Taskinen MR (1997) Lipids and lipoproteins as coronary risk factors in non-insulin-dependent diabetes mellitus. Lancet 350, Suppl. 1, 20-23.

Thomsen C, Rasmussen O, Lousen T, Holst JJ, Fenselau S, Schrezenmeir J \& Hermansen K (1999) Differential effects of saturated and monounsaturated fatty acids on postprandial lipemia and incretin responses in healthy subjects. Am J Clin Nutr 69, $1135-1143$.

Thomsen C, Storm H, Holst JJ \& Hermansen K (2003) Differential effects of saturated and monounsaturated fats on postprandial lipemia and glucagons-like peptide 1 responses in patients with type 2 diabetes. Am J Clin Nutr 77, 605-611.

Tijburg LBM, Mattern T, Folts JD, Weisgerber UM \& Katan MB (1997) Tea flavonoids and cardiovascular disease: a review. Crit Rev Food Sci Nutr 37, 771-785.

Twickler TB, Dallinga-Thie GM, Cohn JS \& Chapman MJ (2004) Elevated remnant-like particle cholesterol concentration. A characteristic feature of the atherogenic lipoprotein phenotype. Circulation 109, $1918-1925$

Vinson JA \& Dabbagh YA (1998) Effect of green and black tea supplementation on lipids, lipid oxidation and fibrinogen in the hamster: mechanisms for the epidemiological benefits of tea drinking. FEBS Lett 433, 44-46.

Yang CS \& Landau JM (2000) Effects of tea consumption on nutrition and health. J Nutr 130, 2409-2412. 